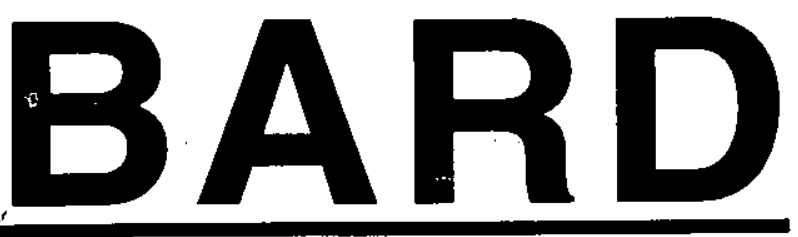

FINAL REPORT

PROJECT NO. US-2062-91

Understanding the Causes and Genetic Effects of Thelytoky in the Aphelinidae: A Key to Improving Biological Control

R.T. Roush, D. Rosen 
Date:

10 June 1996

BARD Project Number: US-2062-91

Project Title:

Understanding the Causes and Genetic Effects of The lytoky in the Aphelinicas: A Key to Improving Biological Contro?

Investigators:

(Irincipal first)

Ricinart I. Roush

David Rosen

Project Start Date: July 1992

Type of Report:

Ist Annual
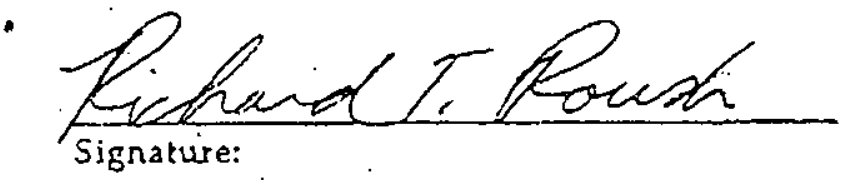

Signature:

Principal Investigator
Affiliated institution:

Curne11 liniversity

The Hebrew University of Jerusalen 2nd Annual __ Final $x x$

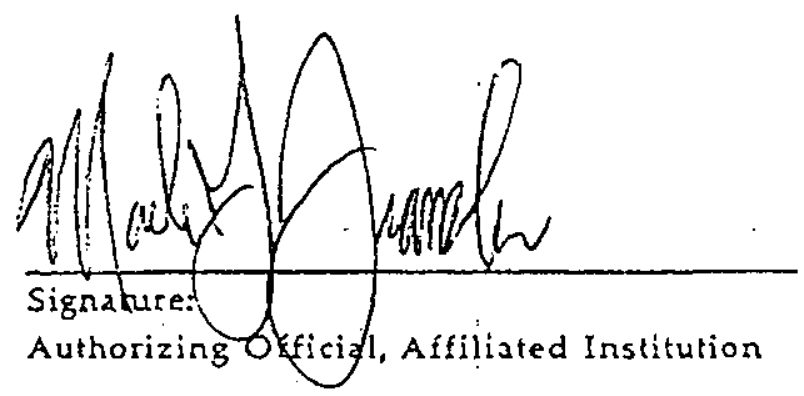


Final Scientific Report

\title{
Understanding the Causes and Genetic Effects of Thelytoky in the Aphelinidae: A Key to Improving Biological Control
}

\author{
Richard T. Roush ${ }^{1}$ and David Rosen ${ }^{2}$, Co-investigators \\ 1'Department of Entomology, Comstock Hall, Cornell University, Ithaca, NY 14853 USA \\ and \\ 2 Department of Entomology, Faculty of Agriculture, The Hebrew University, P.O. Box 12, \\ Rehovot 76100 Israel \\ Table of Contents
}

Abstract 2

Introduction 3

Objectives 4

Relevant data, methodology, results and discussion 5

Description of Cooperation 15

Evaluation of Research Achievement 16

Conclusions 16

References Cited 16

List of Publications from the Project 18

Patents 18 
Roush and Rosen: Thelytoky in the Aphelinidae, p. 2.

Final Scientific Report

\title{
Understanding the Causes and Genetic Effects of Thelytoky in the Aphelinidae: \\ A Key to Improving Biological Control
}

\author{
Richard T. Roush and David Rosen
}

\begin{abstract}
Thelytoky is a type of parthenogenesis whereby females produce only female offspring without the involvement of males, even where males are occasionally produced. In the last few years, strong circumstantial evidence has implied that thelytoky can be caused by microorganisms called Wolbachia in at least some species of wasps. The thelytoky can be "cured" by treatment with antibiotics. Further, Wolbachia-like organisms can be found in microscopic examinations and genetically identified through their DNA.

The aphelinid wasps, and especially species in the genus Aphytis, are among the most important of all classical biological control agents. Aphytis species are critical in the biological control of scale insect pests in commercial orchards and ornamental plantings. About $30 \%$ of Aphytis species are thelytokous, of which we were able to study three in detail. In all three, thelytoky was curable by treatment with antibiotics and Wolbachia were identified morphologically and through their DNA. In contrast, Wolbachia were not detectable in biparental species of Aphytis. Studies of Wolbachia gene sequences obtained from Aphytis showed that they were most closely related to those from a very distantly related wasp, Muscidifurax uniraptor, strongly implying that the Wolbachia can be horizontally transferred. As revealed by electron microscopy, the Wolbachia show a strong association with the nurse and follicle cells of the female wasps.
\end{abstract}


Roush and Rosen: Thelytoky in the Aphelinidae, p. 7

Introduction

Much of the biological control of insect pests is due to parasitic wasps. Most wasps, ants, bees and their relatives (Hymenoptera) reproduce by arrhenotoky, a form of parthenogenesis in which unfertilized eggs give rise to haploid males and fertilized eggs give rise to diploid females. However, complete parthenogenesis, i.e. thelytoky, is also found in : many hymenopterous lineages (Luck et al., 1992). In thelytoky, unfertilized eggs develop into daughters, and these females also produce daughters from unfertilized eggs. Males are. occasionally produced, but are not observed to mate, and mating is not needed for reproduction by females. Consequently, thelytokous lines can persist indefinitely. without sexual reproduction.

Thelytoky was, until recently, assumed to have an exclusively genetic cause in the Hymenoptera. Heat was long known to cause the production of males in thelytokous species, bur Stouthamer et al. (1990) showed for the first time that high temperatures or antibiotic treatments could cause a reversion to arrhenotoky. Most species of parasitic wasps of the genus Trichogramma are arrhenotokous, but thelytokous reproduction also occurs. Stouthamer et al. (1990) exposed 9 lines of 4 species of uniparental Trichogramma to high temperatures and antibiotics. Three antibiotic treatments (rifampicin, tetracycline and sulfamethoxazole) caused a reversion from thelytoky to arrhenotoky, as did the high temperature treatment. These results led to the conclusion that a microorganism was associated with uniparental reproduction in Trichogramma, and this landmark paper inspired the search for such symbionts in other parasitic wasps.

Antibiotic treatments were soon shown to induce the production of males in Encarsia formosa (Zchori-Fein et al., 1992), and important parasite of whiteflies; other Trichogramma (see Rousset et al., 1992); Muscidifurax raptor (Stouthamer et al., 1993), a parasite of filth breeding flies; and other parasitic wasps. As will be discussed further in this report, subsequent work largely by R. Stouthamer and J.H. Werren showed a strong association of microbially-induced thelytoky and bacteria of the genus Wolbachia. Wolbachia species had previously been shown to be strongly associated with the failure of some strains of insects to produce offspring after successful pairings with other strains of the same species (see Rousset et al., 1992 for citations). Strictly speaking, however, because it has not yet been possible to transmit Wolbachia to uninfected hosts, it has not yet been proven conclusively that Wolbachia cause thelytoky.

Among the Hymenoptera, parasites in the family Aphelinidae are especially important in biological control. Thelytokous reproduction occurs sporadically in different groups within the Aphelinidae, especially within the genus Aphytis, which are parasites of scale insect pests. Sixteen species, or about $30 \%$ of Aphytis whose mode of reproduction is known, are thelytokous. Most but not all of these species have related arrhenotokous species or forms 
and the ramifications of thelytokous reproduction for genetic variation and growth of parasite populations, it was important to determine whether uniparental reproduction in Aphytis is associated with the presence of microorganisms, and to try to understand the fundamental effects of those organisms.

\section{Objectives of the Project}

\section{As Stated in the Original Research Proposal}

Primary: Improvement of biological control of armored scale insects by Aphytis species by investigating the causes and genetic effects of thelytoky.

To attain this overall objective, the following specific goals have been set:

1) Determine whether thelytokous Aphytis species can be cured from unisexuality by antibiotic treatments.

2) Investigate the mode of inheritance of genetic traits in the thelytokous species.

3) Assuming that thelytoky can be cured such that uniparental and biparental strains can be established from the same culture:

a) As measured by selection response, compare the adaptability of the arrhenotokous and thelytokous strains.

b) Compare their fitnesses, as measured by reproductive output, under different laboratory and field conditions to determine whether there is a reproductive cost or advantage to thelytoky.

4) In the event that thelytoky cannot be cured, conduct the experiments outlined above on arrhenotokous and thelytokous strains that are as taxonomically similar as possible.

\section{Deviations and Modifications}

Rapid progress was made in this field in 1991-92, largely due to the efforts of $R$. Stouthamer, J. H. Werren, F. Rousset and colleagues. Unknown to us at the time we submitted our grant proposal, there was already research under way that rendered obsolete some of the work we proposed. First, addressing the question of likely causes, bacteria from the genus Wolbachia were found in several wasp species where thelytoky had been shown to be cured by antibiotic treatment (Rousset et al. 1992 [submitted July 1992], Stouthamer et al., 1993 [submitted June 1992]). Second, we learned from a seminar given by Stouthamer that diploidy is restored in Wolbachia-associated thelytokous Trichogramma by gamete duplication (later published by Stouthamer and Kazmer in 1994). When we confirmed that thelytoky in Aphytis was also associated with Wolbachia (as discussed below), this left little doubt as to how genetic traits would be inherited in Aphytis (objective 2).

Based on the implications of these new developments, reviewers' suggestions, and our own early results, we modified some of our plans even within the first year. As described below, we were unable to establish biparental lines from thelytokous lines, in contrast to successes in Trichogramma (Stouthamer et al. 1990a). This rendered objective 3 infeasible, 
and we were therefore unable to directly study the costs to fitness of microbially induced thelytoky. Further, we were unable to obtain from the field arrhenotokous and thelytokous lines that were sufficiently closely related taxonomically to justify valid comparisons of fitness (objective 4). Therefore, we focused our research efforts on the three most important remaining questions about Wolbachia-induced thelytoky: (1) describing how the microorganisms are associated with and may cause thelytoky, and (2) especially when our molecular analysis showed that these had been horizontally transmitted, testing possible routes of transmission, and (3) proving that Wolbachia caused thelytoky by artificial horizontal transmission (i.e., injection).

\section{Relevant Data, Methodology, Results and Discussion Wasps studied}

Three species of Aphytis were successfully studied in detail; Aphytis lingnariensis, Aphytis diaspidis and Aphytis yanonensis. For A. diaspidis and A. yanonensis, we had only uniparental strains, whereas we had both uniparental and biparental "strains" of what were thought to be $A$. lingnanensis. Efforts to study yet another thelytokous species, Aphytis maculicomis, were unsuccessful due to rearing difficulties. In addition, another uniparental species of Aphelinidae, Encarsia formosa, was also used as a control in some experiments. The European spruce sawfly (Gilpinia hercyniae) and the parasitic wasp Venturia $(=$ Nemeritis) canescens, two other uniparental species for which there was no evidence of micro-organismal involvement, were also used as a controls.

Induction of male production by antibiotic treatment.

Following the successes with Trichogramma (Stouthamer et al. 1990a) and Encarsia formosa (Zchori-Fein et al. 1992), a standard preliminary test for whether a case of thelytoky may be due to Wolbachia is to try to cure the infection by feeding a suitable antibiotic to (and/or heat treatment of) the thelytokous female wasps. If a significant increase in male production occurs in the otherwise essentially all female strain, it implies that microorganisms were causing the thelytoky.

For antibiotic treatments, the wasps were typically starved for 24 hours after emergence and then offered antibiotics for 1-2 days mixed with honey (which the wasps readily consume) and food coloring, the latter so that it was possible to observe feeding. With Aphytis lingnanensis, tetracycline and rifampicin were tested at four concentrations $(25,50$, 75 and $100 \mathrm{mg} / \mathrm{ml}$ honey). The progeny ( $\left.\mathrm{F}_{1}\right)$ of $A$. lingnanensis that were fed antibiotics generally showed no higher proportion of males than controls, but these in turn produced about $70-93 \%$ male offspring $\left(\mathrm{F}_{2}\right)$ as opposed to only $10 \%$ in the controls (Zchori-Fein et al. 1995). Fifty $\mathrm{mg} / \mathrm{ml}$ rifampicin was found to be the most effective treatment

Fifty $\mathrm{mg} / \mathrm{ml}$ rifampicin was also used for Aphytis diaspidis. Here the trend was even sharper, with the antibiotic-treated Aphytis diaspidis females producing all female $\mathrm{F}_{1}$ 
offspring, which in turn produced $100 \%$ male $\mathrm{F}_{2}$ offspring. That the effect of antibiotic might not be expressed until the second generation was especially interesting because in all previously successful "curing" experiments, the effect was immediate, that is, males were produced in the $F_{1}$ by the treated females. This suggested that curing could be incomplete and yet the micro-organisms might continue to die off without further treatment, or that substances left by the micro-organisms could have a carry-over effect on eggs in production.

For Aphytis yanonensis, tests were done at $100 \mathrm{mg} / \mathrm{ml}$ rifampicin, and somewhat different results were obtained: $71 \%$ of the $38 \mathrm{~F}_{1}$ offspring were males and all $50 \mathrm{~F}_{2}$ offspring were males. In untreated controls, only 5 of 62 offspring were males. It is not clear why $A$. yanonensis responded more quickly and fully to the antibiotic treatment, although it could be due to differences in the concentration (and batch) of the antibiotic. In terms of gene sequences, the Wolbachia isolated from all three species seemed very similar, as will be discussed later in this report.

In contrast to the results with Aphytis, there was no significant increase of percentage of male sawflies in $F_{1}$ or $F_{2}$ offspring after extensive treatments with rifampicin, and no production of males whatsoever in Venturia canescens. For the spruce sawfly, where the adults would not feed, rifampicin was given to larvae by force feeding, painting on spruce foliage, and injection into larvae. These and subsequent PCR experiments (see below) confirmed that not all thelytoky in the Hymenoptera is associated with Wolbachia.

\section{Visual confirmation of Wolbachia-like organisms.}

With appropriate stains and under light microscopy, Stouthamer and Werren (1993) provided evidence of symbionts in 13 Trichogramma lines that exhibited revertible parthenogenesis (i.e., can be "cured" by antibiotic). Such symbionts were not found in the eggs of 6 field-collected arrhenotokous lines, 4 non-revertible parthenogenetic lines or in 3 arrhenotokous lines derived from thelytokous lines produced by antibiotic treatments. We were similarly able to confirm by both light and electron microscopy that Wolbachia-like microorganisms were present in thelytokous but not "cured" or arrhenotokous A. lingnanensis (Zchori-Fein et al. 1994). Ovaries of Encarsia formosa were also examined and found to contain microorganisms with the same ultrastructure. Further observations under microscopy will described later in this report.

\section{Use of males from cured females to develop new biparental lines for fitness studies}

As one of the original objectives of our grant proposal was to see if cured wasps could contribute to an arrhenotokous (sexually reproducing) strain, males produced from antibiotic treatments were placed with females from antibiotic treated and untreated mothers, and the colony observed for sexual reproduction.

Sexual reproduction was accomplished in four similarly treated species of Trichogramma (Stouthamer et al. 1990a), and in other species where heat was used to induce 
the males (Legner 1985), but not in Aphytis. For all three species of Aphytis, males were mated to $F_{1}$ females from antibiotic treated mothers under various environmental conditions to encourage mating and occasionally were seen to mate. However, no sperm were found in the spermathecae of mated females, which all produced exclusively all-male broods, as is expected for unmated arrhenotokous females (which can lay only unfertilized eggs).

The most detailed and compelling results were obtained with $A$. lingnanensis, for which two morphologically indistinguishable lines are maintained at the Faculty of Agriculture in Rehovot: One strain is uniparental and the other is biparental, so in contrast to the other species examined, it was possible to mate males from antibiotic-treated uniparental females with females from the biparental line, following a procedure used by Stouthamer et al. (1990b).

Males of the uniparental line inseminated about $54 \%$ of their own females (Table 1 , UXU cross), which did not differ significantly from the percent insemination seen in the control (biparental females with biparental males, BXB). However, although sperm from the uniparental line appeared normal (Figure 1), all of the females mated by these males produced exclusively male progeny, indicating that fertilization did not occur.

Table 1. Presence of sperm in the spermathecae of Aphytis lingnanensis females mated

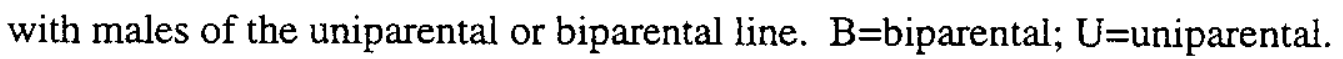

\begin{tabular}{cccc}
$\begin{array}{c}\text { crosses } \\
\text { female X male }\end{array}$ & $\begin{array}{c}\text { \# of spermatheca } \\
\text { examined }\end{array}$ & $\begin{array}{c}\text { \# of full } \\
\text { spermatheca }\end{array}$ & \multicolumn{2}{c}{$\begin{array}{c}\text { mean \% of full } \\
\text { spermathecae (SE) }\end{array}$} \\
B X B & 29 & 16 & 67 \\
U X U & 28 & 15 & $54(11)$ \\
B X U & 38 & 1 & $2.8(9.3)$ \\
U X B & 35 & 0 & $0.0(0)$
\end{tabular}

In crosses between strains, only one out of 38 spermathecae examined was found to contain sperm (Table 1, BXU cross). The differences between within (UXU and BXB) and between (BXU and $\mathrm{UXB}$ ) strain crosses was highly significant $(F=16, p<0.001)$, suggesting that the two strains have significant pre-zygotic (or pre-mating) barriers and should be considered separate species.

The precise cause of the failure of antibiotic treated Aphytis to reproduce biparentally was not resolvable. It was as if the species have been uniparental so long that the females have lost the ability to use the sperm (presumably due to selection against a disused function). Perhaps the males have not lost the ability to produce sperm simply because they don't reproduce, such that selection is not operating on sperm production. However, it is also possible that something was wrong with the sperm. In any case, our results contrast with those with Trichogramma (Stouthamer et al. 1990a, 1990b), and suggest that the association of Wolbachia with Aphytis may be older than that for Trichogramma. 


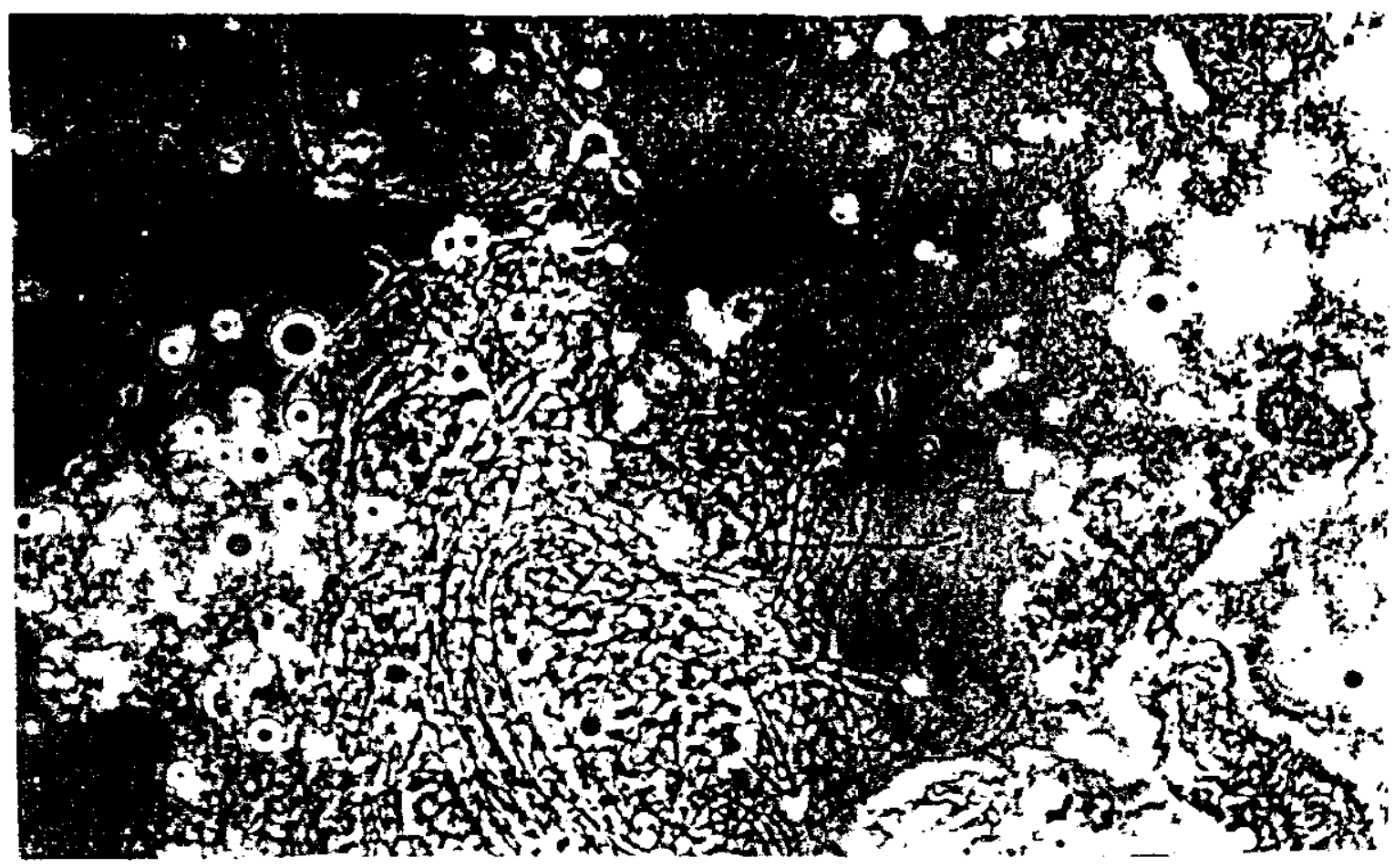

Figure 1. Squash of reproductive tract of an antibiotic-induced Aphytis lingnanensis male, showing sperm (x1000).

In summary, that it is possible to induce the production of males in a thelytokous Aphytis line by antibiotics suggests that microorganisms play a role in determining the mode of reproduction in these wasps. However, it was not possible to establish a biparental line of any Aphytis species. Similar results were obtained with Encarsia formosa (Zchori-Fein et al. 1992). Further, in contrast to the case with Trichogramma, the one morphologically identical biparental strain that was available proved to be reproductively incompatible. Thus, we were not able to execute comparative fitness studies.

In principle, a parasitic wasp that reproduces only daughters could produce twice as many offspring that can attack and kill hosts (which the males cannot) as a wasp that produces both sexes, depending on sex ratio and assuming that total number of offspring is the same. For that reason, and because uniparental strains are easier to colonize, thelytokous strains are attractive in biological control (Aeschlimann 1990). However, among Encarsia formosa treated with tetracycline, antibiotic-treated females produced about twice as many progeny than did the untreated control (Zchori-Fein et al. 1992), suggesting that antibiotic treatment was, in effect, controlling a disease organism. Other studies on strains with cured thelytoky have yielded results that may have been somewhat confounded by the antibiotic used. Antibiotic treatments of uniparental lines of Trichogramma deion and T. pretiosum resulted in an increase in the production of progeny, and in two of three cases arrhenotokous females produced more females than their thelytokous counterparts when host availability was not limited (Stouthamer and Luck 1993). In contrast, treatment of E. formosa with rifampicin significantly reduced 
Roush and Rosen: Thelytoky in the Aphelinidae, p. 9

reproduction relative to the untreated control (Stouthamer et al. 1994). There were no significant differences in fecundity between rifampicin-treated and untreated Muscidifurax uniraptor (a uniparental species) (Stouthamer et al. 1994).

\section{Confirmation and identification of Wolbachia via PCR amplification of eubacterial 16S rDNAs}

Following the use of PCR to demonstrate via sequence analysis of bacterial $16 \mathrm{~S}$ ribosomal DNA that Wolbachia were associated with thelytoky (Rousset et al. 1992, Stouthamer et al. 1993), PCR has become a primary diagnostic tool for investigating the probable cause of thelytoky in wasps. In addition, analysis of the DNA sequences obtained allows one to investigate the phylogenetic relationships of the Wolbachia involved, and investigate the possibility of horizontal transfer of the Wolbachia between wasp lineages. (We thank R. Stouthamer at the Agricultural University of the Netherlands at Wageningen for early discussions on this phase of the project.)

The details for the reaction procedures used for Aphytis lingnanensis and A. diaspidis are given in Zchori (1995) and Zchori et al. (1995) and were varied only slightly for $A$. yanonensis. In all cases, the primers were designed to be specific for Wolbachia pipientis in wasps; forward primer: 5'- TTGTAGCTTGCTATGGTATAACT; reverse primer: 5'GAATAGGTATGATTTCCATGT. The primers were based on those of O'Neill et al. (1992), except that we modified our forward primer with a $\mathrm{T}$ instead of $\mathrm{C}$ in position 8 , and the reverse primer with a $C$ instead of $T$ in position 17 , based on the sequence published by Breeuwer et al. 1992 from Nasonia.

A $16 \mathrm{~S}$ rDNA sequence of the proper length (1000 base pairs) was obtained from all three thelytokous species of Aphytis studied, but not from wasps from cured uniparental strains or two biparental lines (Aphytis lingnanensis and Aphytis melinus) used as controis (Zchori- Fein et al. 1995 and unpublished data). This confirmed the association of Wolbachia with thelytoky in Aphytis. Similarly, a fragment of the correct size was obtained from $E$. formosa, the other thelytokous aphelinid thought to be infected with Wolbachia (Zchori-Fein et al. 1992). In contrast, no fragments were obtained from the uniparental European spruce sawfly, Venturia canescens or two other uniparental strains, Cape honeybees (Apis mellifera from South Africa) and Andricus quercuscalifornicus (another bee). In fact, no signals could be obtained from the fater four uniparental hymenopteran species even when the more general Wolbachia-specific primers were used.

Combined with the evidence that antibiotic treatment induced the production of males, v the signal of about 1000 bases gives a strong indication that Wolbachia is responsible for parthenogenetic reproduction in all three thelytokous strains of Aphytis tested as well as the Encarsia.

The sequences of the 16S rDNA of Wolbachia from the uniparental Aphytis lingnanensis, A. diaspidis (deposited in Gene Bank under X87406 and X87407), and $A$ : yanonensis were almost identical across the regions sequenced, yet considerably different than 
Cytological observations of Nasonia revealed that eggs produced in incompatible crosses are normally fertilized, but syngamy of maternal and paternal pronuclei is aborted, resulting in haploid embryos (Breeuwer and Werren 1990). In Drosophila, where haploid embryos can't develop, developmental defects in cleavage nuclei result in sterility (O'Neill and Karr 1990). In contrast, the cytogenetic mechanism by which diploidy is restored in Wolbachia-associated thelytokous Trichogramma was shown to be gamete duplication (Stouthamer and Kazmer 1994). How a virtually identical microorganism can have these two completely different mechanisms for chromosomes manipulation is still an open question. One possibility is that the 16S rDNA sequence analysis does not provide a true phylogeny for these rickettsia-like symbionts. This possibility has been at least partially excluded by Rousset et al. (1992), who obtained essentially the same phylogeny based on 23S rDNA analysis. A second explanation is that different Wolbachia strains contain some virus or plasmid which is responsible for the chromosomal alteration in the host. No evidence for viruses was found in our electron microscopic studies (Zchori-Fein et al. 1994), but we cannot rule out that possibility.

A closer examination of the relationships among the Wolbachia that cause thelytoky confirm the close relationship between those from $M$. uniraptor and Aphytis (lower clade, Figure 3). Given the very distant relationships of the two wasp genera (different insect families, with Muscidifurax in the Pteromalidae) and even their hosts and habitats (filth breeding fly pupae and scale insects on plants, respectively), it seems stunning that these two insect genera would share a very similar bacterium, and strongly suggests horizontal transfer. Assuming substitution rates similar to those estimated in other prokaryotes, the common ancestor of examined Wolbachia lived about 25-100 million years ago. This makes them much younger than any common ancestor of the hosts ( $>300$ million years) (Moran and Baumann 1994). The investigation of horizontal transfer became another major thrust of our research effort.

How can the microorganisms cause thelytoky? Clues from antibiotic treatments

As noted above, males often appear only in the $\mathrm{F}_{2}$ of antibiotic-treated uniparental Aphytis. However, no microorganisms can be seen in electron micrographs even of the $\mathrm{F}_{1}$ of rifampicin-fed A. lingnanensis (Zchori-Fein et al. 1994). These results raise the question of correlation between the actual presence of Wolbachia and its influence on the host. Wolbachiaspecific primers were used to test for the presence of the symbiont in the F1 of rifampicin-fed females.

In the absence of hosts for oviposition, a strong PCR band for the Wolbachia $16 \mathrm{~S}$ rDNA was detected in A. lingnanensis females even 7 days after antibiotic treatment (ZchoriFein et al. 1995). A much weaker PCR product was obtained up to three days post-treatment when the wasps were permitted to oviposit. No detectable band was obtained in the daughters $\left(F_{1}\right)$ of treated females, consistent with observations from electron microscopy. 

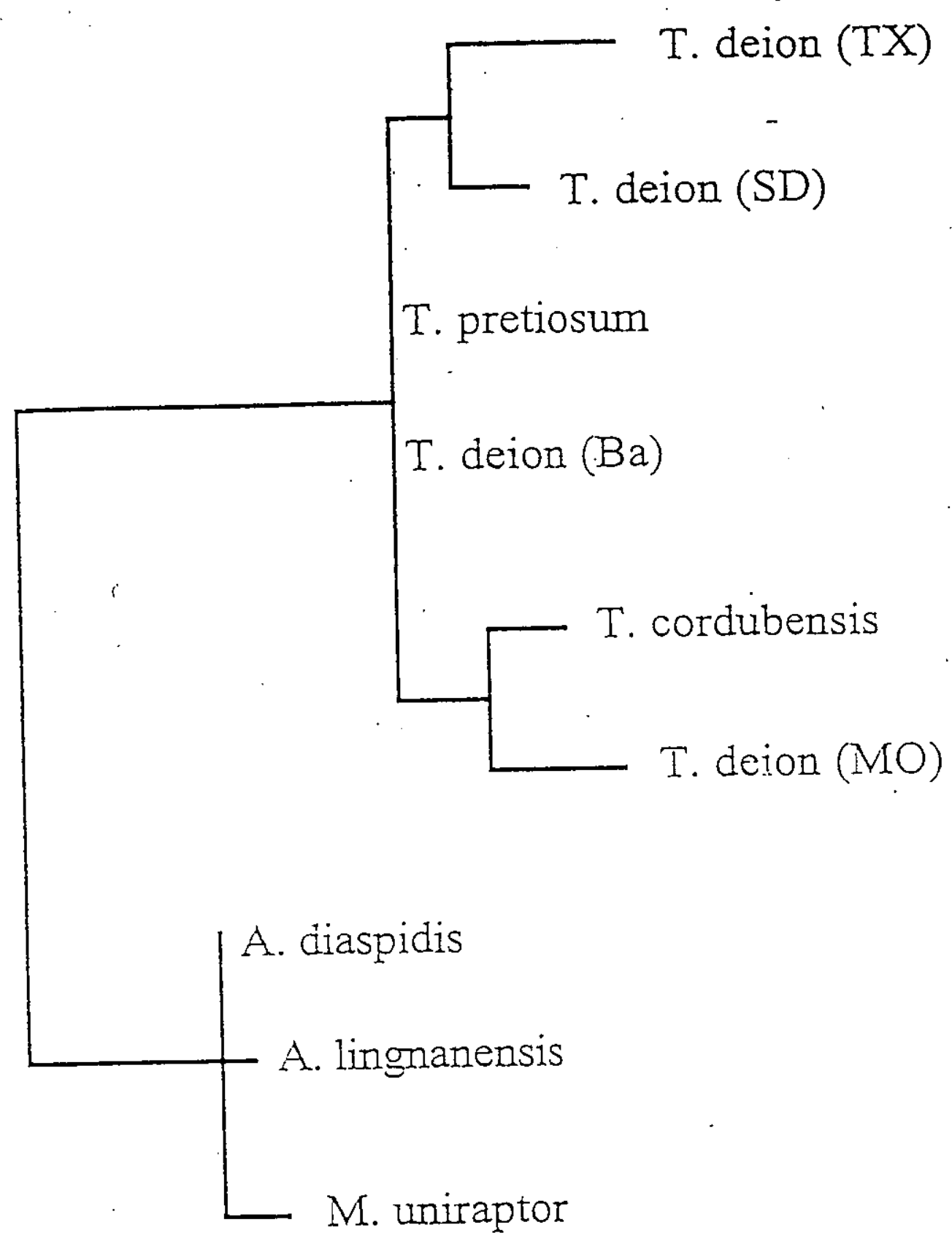

Figure 3. A most parsimonious phylogenetic tree of parthenogenesis microorganisms based on 16S rDNA sequences. The tree was constructed using branch-and-bound method of PAUP, with Ehrlichia platys as an outgroup ( $\mathrm{A}=$ Aphytis; $\mathrm{M}=$ Muscidifurax; $\mathrm{T}=$ Trichogramma; collection site abbreviations in parentheses are those of Stouthamer et al. [1993]). 
Roush and Rosen: Thelytoky in the Aphelinidae, p. 13

Although Wolbachia were detectable in antibiotic-fed wasps that were allowed to lay eggs, that signal was much weaker than in wasps that were presumably forced to resorb their eggs. This difference in signals might best be explained by the presence of non-degraded DNA of dead Wolbachia in the ovaries of the wasps which were not allowed to oviposit, because the eggs when laid seem to produce daughters without Wolbachia (Zchori-Fein et al. 1995). However, the fact that at least in A. lingnanensis and A. diaspidis the $\mathrm{F}_{1}$ offspring of antibiotic-treated females were mainly females suggests that thelytokous reproduction is induced by an unknown factor released by the symbiont.

How can the microorganisms cause thelytoky? Localisation of Wolbachia in ovaries and eggs

Summarizing the results of a great deal of transmission electron, confocal and two photon laser scanning microscopy, Wolbachia were found only in the gonads of females from the untreated uniparental lines, and mainly in nurse cells and eggs. Within the eggs, they were always found concentrated in the vicinity of the egg nuclei (similar to observations for Wolbachia involved in mating [cytoplasmic] incompatibilities [O'Neill and Karr 1990]), and usually on one side rather than evenly distributed around the nucleus. When two neighboring nuclei were observed, the symbionts were located between them (Figure 4). Some symbionts were found in close association with the nucleus, their membranes virtually touching that of the nucleus, and others were found closely crowded around the nuclear organizing center (ZchoriFein 1995). This placement of the microorganisms would surely assist them in altering the function of chromosomes in their hosts.

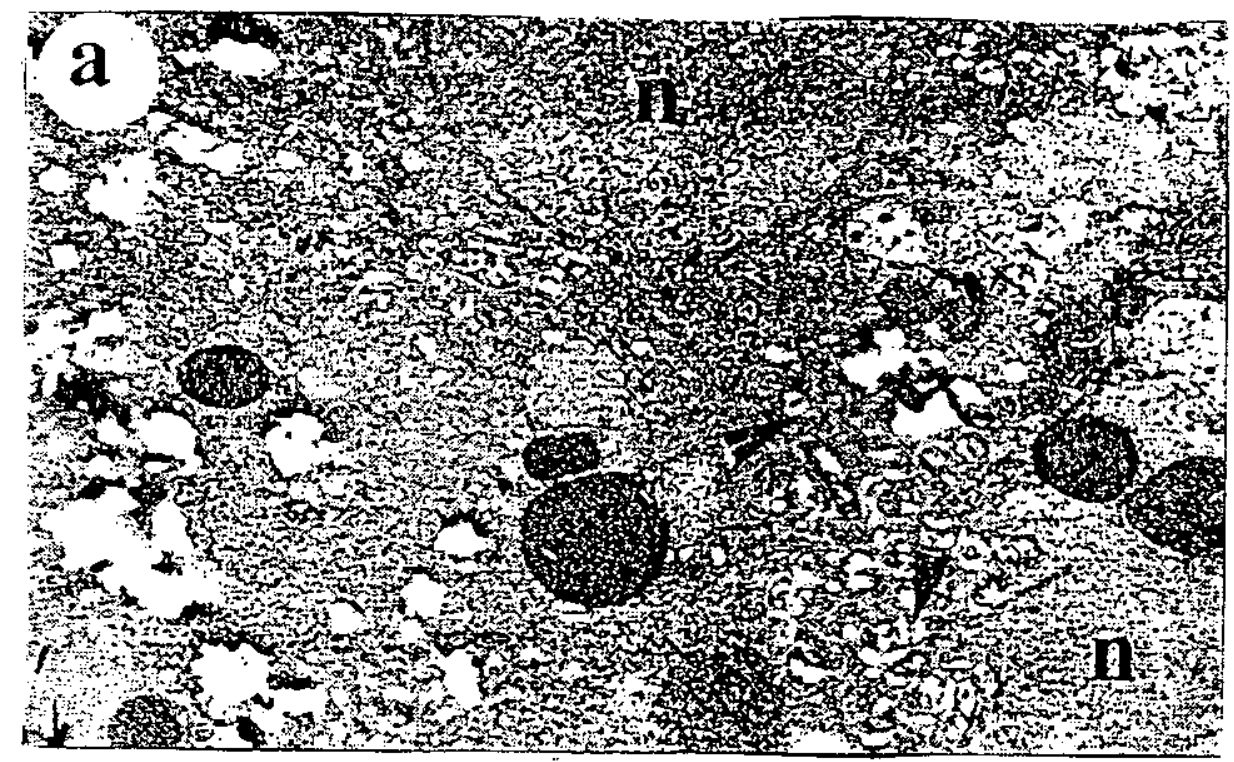

Figure 4. Wolbachia in the vicinity of nuclei in eggs of uniparental Aphytis lingnanensis. (a) 0-2 hold egg, with two nuclei. The symbionts are concentrated between the two nuclei $(X 5,000)$. (b) Higher magnification of (a) $(X 6,600)$. $n=$ nucleus; arrowheads point to Wolbachia. 
How are Wolbachia transmitted between species? Tests of hosts

One of the most intriguing questions about. Wolbachia is how it is acquired. Given the wide range of insects in which the Wolbachia have been found, including many where no effects had been previously noted (O'Neill et al. 1992), it seems possible that parasitic wasps acquire the Wolbachia through parasitism or feeding on other insects. For this reason, we used PCR to test large numbers of the hosts of Aphytis for the presence of the Wolbachia involved in thelytoky.

We tested all of the host insects used at both labs, including the seven armored scale insects, Hemiberlesia lataniae, Aspidiotus nerii, Aonidiella aurantii, Parlatoria oleae and Diaspis echinocacti, Quadraspidiotus pernieiosupi, and a uniparental strain of A. nerii, plus two hosts of Encarsia, Bemisia tabaci (sweetpotato whitefly) and the greenhouse whitefly Trialeurodes vaporariorum. In addition we looked for Wolbachia among species that live or feed on the cactus scale Diaspis echinocacti. Cactus pads were collected from campuses of the Faculty of Agriculture and the Weizmann Institute, Rehovot, and from BetElazari, near Rehovot, and placed in dark emergence cages. The arthropods that emerged were Marietta javensis, Habrolepis sp., Rhizobius sp., Hemisarcoptes coccophagus, Aphytis sp. and Psocoptera. Although we obtained the occasional and tantalizing positive band from some experiments, none of the results were repeatable, and we were forced to conclude that these hosts and associated insects were not a source of the same Wolbachia as found in Aphytis.

\section{Proof of the causative agent: Culturing Wolbachia.}

Due to their extremely fastidious nature, it has not been possible to isolate Wolbachia and grow them in pure culture or to convert an arrhenotokous strain to thelytoky through infection. With help from the Insect Biocontrol Lab, USDA, Beltsville, Maryland, we tried extensively to culture Wolbachia from the Drosophila simulans, Riverside strain (a strain known to carry Wolbachia, O'Neill and Karr 1990) and Aphytis yanonensis by inoculating insect ovaries and homogenizations (filtered through $0.45 \mathrm{um}$ Millex filters) into 12 different Lepidopteran, Coleopteran and Hymenopteran insect cell lines (Hackett et al. 1986). No Wolbachia-like organisms were detected in the cells of samples inoculated with insect ovaries by using fluorescent stain (Hoechst 33258) after three weeks.

\section{Proof of the causative agent: Injection experiments}

It has been possible to transfer cytoplasmic mating incompatibility between strains of Nasonia (Williams et al. 1993); although these authors did not identify the causative agent, other studies indicate that Wolbachia might be responsible (Breeuwer et al. 1992). It has also been possible to transfer the Wolbachia responsible for mating incompatibilities of Drosophila between strains and species (Boyle et al. 1993, Braig et 
al. 1994). Given the small size of Aphytis, and the apparently close relationship of the Aphytis from Wolbachia to that from Muscidifurax uniraptor, we attempted injections into its sibling species Muscidifurax raptor. To give ourselves the maximum chance of a result, we used preparations from $A$. yanonensis, Encarsia formosa, Muscidifurax uniraptor, and Drosophila simulans, Riverside strain . Uninfected Drosophila were also used as controls.

Rather than try to inject directly into wasp pupae (where we had high control mortality) or their eggs (which had proven extremely difficult) we injected into the abdomens of adult females. This seemed reasonable since the Wolbachia appeared concentrated around the female reproductive tissues. Parasitized house fly pupae were held in individual gel capsules to isolate individuals so unmated female wasps would remain virgins. An electronic micro-injection apparatus with a joy stick for added control was used for the injections. Glass needles were pulled and then filled with Wolbachia preparations or control solutions. The needle was inserted between the 4th and 5th or 5th and 6th segments at a diagonal direction aiming for the nurse cells of the female. The solution was injected into the wasp by syringe.

For injections from Drosophila simulans, ovaries of the flies were dissected into a petri dish of TES Ringer solution. The ovaries were then macerated manually with a grinding rod and micro centrifuged for a few seconds to separate the larger particles from the supernatant, which was used in injecting the female wasp. A total of 99 wasps were injected, 50 with solutions from infected flies and 49 with solutions from uninfected flies. Of those, 41 and 49 wasps, respectively, survived injection.

When injected with Encarsia and Aphytis preparations, the entire wasp pupa was used in the preparation at concentrations of 2 pupae of Encarsia /50 $\mu$ l of TES ringer and about 1225 individuals of Aphytis /50 $\mu$ l TES ringer. The samples were then homogenized with a mechanical grinder, centrifuged and then the supernatant injected. A total of 53 mated female wasp, were injected with Encarsia, with 48 wasps injected with TES ringer solution as controls. All control-injected wasps survived. Only 8 of 53 Encarsia-injected wasps survived and then for only 3 days, suggesting that there was something toxic in Encarsia preparations. A total of 33 mated females were injected with Aphytis and 39 injected with ringer solution.

Hundreds of wasps were reared in individual female lines from these injected females, with no differences observed in the sex ratios of the offspring or mating compatibilities over three generations.

\section{Description of Cooperation}

Through frequent communication via e-mail and FAX, both sides kept updated on the other's progress and developed a division of research emphases that was appropriate to the skills and interests of each side. The visit of Roush to Israel in August 1993 and Zchori-Fein to New York in August and September of 1994 was very important and effective for sharing skills and ideas. During her visit to Cornell University, Zchori-Fein was able to use a state-ofthe-art experimental two photon laser scanning microscope in the Laboratory of Professor Watt 
W. Webb in the Department of Applied Physics at Cornell for examination of Wolbachia. If only the organisms studied had been so cooperative!

\section{Evaluation of Research Achievement}

Consistent with the original research objectives, we documented that Wolbachia were responsible for thelytoky in Aphytis and investigated the causes of the thelytoky at the cellular level. We provided the first strong evidence for the horizontal transfer of thelytoky-inducing Wolbachia (see Figure 3). We described in detail the localization of Wolbachia within the wasp, and showed that the Wolbachia were found only in the reproductive tissues of the females and mainly in nurse cells and eggs. We also provided circumstantial evidence from microscopic and PCR studies of antibiotic treated females and their cured daughters that thelytokous reproduction is induced by àn unknown factor released by the Wolbachia, rather than the physical presence of the Wolbachia itself. One student, Einat Zchori-Fein, completed a $\mathrm{PhD}$, and another, Yuval Gottlieb, has completed much of the research for a masters degree. Finally, we showed that the uniparental line previously called Aphytis lingnanensis should be considered a new species and assigned a new name.

\section{Conclusions}

The exact means by which Wolbachia is able to cause thelytoky and can be transmitted from one species to the next remains a mystery. On the basis of our injection work and the survey of hosts, plus the observation that most Aphytis are still biparental, it is tempting to speculate that acquiring a Wolbachia infection is a rare event.

Because uniparental forms of parasitoids do not require mates, it has been argued that thelytokous strains should be sought out and used in biological control to insure rapid establishment (Aeschlimann 1990). It has now become clear, however, that many strains are thelytokous because of Wolbachia, which could restrict fitness and genetic recombination and therefore might be considered a pathogen. While we were not able to directly test whether Wolbachia cause fitness costs to the wasps that carry them, other work suggests that thery do. Further, we did find that there is evidence for horizontal transfer of Wolbachia, which suggests that the deliberate importation of thelytokous forms not only runs the risk of loading fitness costs on the specific biocontrol effort, there is also the chance of transmission of these costs to other agents. Even if transmission is a rare event, the chances may be high once the parasites reach large population sizes. For these reasons, it seems prudent to avoid release of thelytokous forms, especially where arrhenotokous forms are available.

\section{References Cited}

Aeschlimann, J.-P. 1990. Simultaneous occurrence of thelytoky and bisexuality in hymenopteran species, and its implications for the biological control of pests. Entomophaga 35: 3-5. 
al. 1994). Given the small size of Aphytis, and the apparently close relationship of the Aphytis from Wolbachia to that from Muscidifurax uniraptor, we attempted injections into its sibling species Muscidifurax raptor. To give ourselves the maximum chance of a result, we used preparations from A. yanonensis, Encarsia formosa, Muscidifurax uniraptor, and Drosophila simulans, Riverside strain. Uninfected Drosophila were also used as controls.

Rather than try to inject directly into wasp pupae (where we had high control mortality) or their eggs (which had proven extremely difficult) we injected into the abdomens of adult females. This seemed reasonable since the Wolbachia appeared concentrated around the female reproductive tissues. Parasitized house fly pupae were held in individual gel capsules to isolate individuals so unmated female wasps would remain virgins. An electronic micro-injection apparatus with a joy stick for added control was used for the injections. Glass needles were pulled and then filled with Wolbachia preparations or control solutions. The needle was inserted between the 4 th and 5 th or 5 th and 6 th segments at a diagonal direction aiming for the nurse cells of the female. The solution was injected into the wasp by syringe.

For injections from Drosophila simulans, ovaries of the flies were dissected into a petri dish of TES Ringer solution. The ovaries were then macerated manually with a grinding rod and micro centrifuged for a few seconds to separate the larger particles from the supernatant, which was used in injecting the female wasp. A total of 99 wasps were injected, 50 with solutions from infected flies and 49 with solutions from uninfected flies. Of those, 41 and 49 wasps, respectively, survived injection.

When injected with Encarsia and Aphytis preparations, the entire wasp pupa was used in the preparation at concentrations of 2 pupae of Encarsia /50 $\mu 1$ of TES ringer and about 1225 individuals of Aphytis $150 \mu$ TES ringer. The samples were then homogenized with a mechanical grinder, centrifuged and then the supernatant injected. A total of 53 mated female

$\times \quad$ wasp, were injected with Encarsia, with 48 wasps injected with TES ringer solution as controls. All control-injected wasps survived. Only 8 of 53 Encarsia-injected wasps survived and then for only 3 days, suggesting that there was something toxic in Encarsia preparations. A total of 33 mated females were injected with Aphytis and 39 injected with ringer solution.

Hundreds of wasps were reared in individual female lines from these injected females, with no differences observed in the sex ratios of the offspring or mating compatibilities over three generations.

\section{Description of Cooperation}

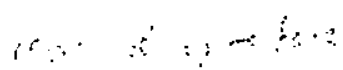

Through frequent communication via e-mail and FAX, both sides keptupdated, on the other's progress and developed a division of research emphases that was appropriate to the skills and interests of each side. The visit of Roush to Israel in August 1993 and Zchori-Fein to New York in August and September of 1994 was very important and effective for sharing skills and ideas. During her visit to Cornell University, Zchori-Fein was able to use a state-ofthe-art experimental two photon laser scanning microscope in the Laboratory of Professor Watt 
W. Webb in the Department of Applied Physics at Cornell for examination of Wolbachia. If only the organisms studied had been so cooperative!

\section{Evaluation of Research Achievement}

Consistent with the original research objectives, we documented that Wolbachia were responsible for thelytoky in Aphytis and investigated the causes of the thelytoky at the cellular level. We provided the first strong evidence for the horizontal transfer of thelytoky-inducing Wolbachia (see Figure 3). We described in detail the localization of Wolbachia within the wasp, and showed that the Wolbachia were found only in the reproductive tissues of the females and mainly in nurse cells and eggs. We also provided circumstantial evidence from microscopic and PCR studies of antibiotic treated females and their cured daughters that thelytokous reproduction is induced by an unknown factor released by the Wolbachia, rather than the physical presence of the Wolbachia itself. One student, Einat Zchori-Fein, completed a $\mathrm{PhD}$, and another, Yuval Gottlieb, has completed much of the research for a masters degree. Finally, we showed that the uniparental line previously called Aphytis lingnanensis should be considered a new species and assigned a new name.

\section{Conclusions}

The exact means by which Wolbachia is able to cause thelytoky and can be transmitted from one species to the next remains a mystery. On the basis of our injection work and the survey of hosts, plus the observation that most Aphytis are still biparental, it is tempting to speculate that acquiring a Wolbachia infection is a rare event.

Because uniparental forms of parasitoids do not require mates, it has been argued that thelytokous strains should be sought out and used in biological control to insure rapid establishment (Aeschlimann 1990). It has now become clear, however, that many strains are thelytokous because of Wolbachia, which could restrict fitness and genetic recombination and therefore might be considered a pathogen. While we were not able to directly test whether Wolbachia cause fitness costs to the wasps that carry them, other work suggests that thery do. Further, we did find that there is evidence for horizontal transfer of Wolbachia, which suggests that the deliberate importation of thelytokous forms not only runs the risk of loading fitness costs on the specific biocontrol effort, there is also the chance of transmission of these costs to other agents. Even if transmission is a rare event, the chances may be high once the parasites reach large population sizes. For these reasons, it seems prudent to avoid release of thelytokous forms, especially where arrhenotokous forms are available.

\section{References Cited}

Aeschlimann, J.-P. 1990. Simultaneous occurrence of thelytoky and bisexuality in hymenopteran species, and its implicátions for the biological control of pests. Entomophaga 35: 3-5. 
Buyle, L., S. L. O'Neill, H. M. Robertson, and T. L. Karr. 1993. Interspecific and intraspecific horizontal transfer of Wolbachia in Drosophila. Science 260: 1796-99.

Braig, H. R., H. Guzman, R. B. Tesh, and S. L. O'Neill. 1994. Replacement of the natural Wolbachia symbiont of Drosophila simulans with a mosquito counterpart. Nature 367: 453-455.

Breeuwer, J. A. J., and J. H. Werren. 1990. Microorganisms associated with chromosome destruction and reproductive isolation between two insect species. Nature 346: 558-560.

Breeuwer, J. A. J., R. Stouthamer, S.M. Barns, D.A. Pelletier, W. G. Weisburg, and J. H. Werren. 1992. Phylogeny of cytoplasmic incompatibility microorganisms in the parasitoid wasp genus Nasonia (Hymenoptera: Pteromalidae) based on $16 \mathrm{~S}$ ribosomal DNA sequences. Insect Molec. Biology 1: 25-36.

Hackett, K. J., D. E. Lynn, D. L. Williamson, A. S. Ginsberg, and R. F. Whitcomb. 1986. Cultivation of the Drosophila sex-ratio spiroplasma. Science 232: 1253-54.

Zchori-Fein, E., R. T. Roush, and M. Hunter. 1992. Male production induced by antibiotic treatment in Encarsia formosa, an asexual species. Experientia 48: 102-105.

Legner, E. F. 1985. Effects of scheduled high temperature on male production in thelytokous Muscidifurax uniraptor. Canadian Entomologist 117: 383-389.

Moran, N. and P. Baumann. 1994. Phylogenetics of cytoplasmically inherited microorganisms of arthropods. TREE 9: 15-20.

O'Neill, S. L., and T. L. Karr. 1990. Bidirectional incompatibility between conspecific populations of Drosophila simulans. Nature 348: 178-180.

O'Neill, S. L., R. Giordano, A.M.E. Colbert, T. L. Karr, and H. M. Robertson. ' 1992. $16 \mathrm{~S}$ rRNA phylogenetic analysis of the bacterial endosymbionts associated with cytoplasmic incompatibility in insects. Proc. Natl. Acad. Sci. USA 89: 2699-702.

Rosen, D., and P. DeBach. 1979. Species of Aphytis of the World (Hymenoptera: Aphelinidae). Israel Universities Press, Jerusalem, and W. Junk, The Hague (Series Entomologica, Vol. 17).

Rousset, F., D. Bouchon, B. Pintureau, P. Juchault, P., and M. Solignac. 1992. Wolbachia endosymbionts responsible for various alterations of sexuality in arthropods. Proc. $R$. Soc. Lond. B 250: 91-98.

Stouthamer, R., and D. J. Kazmer. 1994. Cytogenetics of microbe-associated parthenogenesis and its consequences for gene flow in Trichogramma wasps. Heredity 73: 317-327.

Stouthamer, R. and R. F. Luck. 1993. Influence of microbe-associated parthenogenesis on the fecundity of Trichogramma deion and T. pretiosum. Entomol. Exp. Appl. 67: 183-192.

Stouthamer, R. and J. H. Werren. 1993. Microorganisms associated with parthenogenesis in wasps of the genus Trichogramma. J. Invert. Pathol. 61: 6-9. 
Stouthamer, R., R. F. Luck and W. D. Hamilton. 1990a. Antibiotics cause parthenogenetic Trichogramma (Hymenoptera: Trichogrammatidae) to revert to sex. Proc. Nat. Acad. Sci. 87: 2424-2427.

Stouthamer, R., J. D. Pinto, G. R. Platner and R. F. Luck. 1990b. Taxonomic status of thelytokous forms of Trichogramma (Hymenoptera: Trichogrammatidae). Ann. Entomol. Soc. Amer. 83: 475-481.

Stouthamer, R., Breeuwer, J.A.J., R. F. Luck, and J. H. Werren. 1993. Molecular identification of microorganisms associated with parthenogenesis. Nature 361: 66-68.

Stouthamer, R., S. Luko, and F. Mak. 1994. Influence of parthenogenesis Wolbachia on host fitness. Norw. J. Agricultural Sciences. Suppl 16: 117-122.

Williams, E. H., Fields, S., and G. B. Saul II. 1993. Transfer of incompatibility factors between stocks of Nasonia (=Mormoniella) vitripennis. J. Invert. Pathol. 61: 206-10.

Zchori-Fein, E. 1995. Association between parasitic wasps of the genus Aphytis (Hymenoptera: Aphelinidae) and parthenogenesis-inducing symbionts. $\mathrm{PhD}$ thesis, Hebrew University of Jerusalem.

Zchori-Fein, E., R. T. Roush, and M. Hunter. 1992. Male production induced by antibiotic treatment in Encarsia formosa, an asexual species. Experientia 48: 102-105.

Zchori-Fein, E., D. Rosen, and R. T. Roush. 1994. Microorganisms associated with thelytoky in Aphytis lingnanensis Compere (Hymenoptera: Aphelinidae). Int. J. of Insect Morphology and Embryology 23: 169-172.

Zchori-Fein, E., O. Faktor, M. Zeidan, Y. Gottlieb, H. Czosnek, and D. Rosen. 1995. Parthenogenesis-inducing microorganisms in Aphytis (Hymenoptera:

Aphelinidae). Insect Molecular Biology 4:173-178.

\section{List of Publications From This Research}

Argov, Y., E. Zchori-Fein and D. Rosen. Biosystematic studies in the Aphytis lingnanensis complex. Israel $\mathrm{J}$. Entomol. (in press).

Zchori-Fein, E. 1995. Association between parasitic wasps of the genus Aphytis (Hymenoptera: Aphelinidae) and parthenogenesis-inducing symbionts. PhD thesis, Hebrew University of Jerusalem.

Zchori-Fein, E., D. Rosen, and R. T. Roush. 1994. Microorganisms associated with thelytoky in Aphytis lingnanensis Compere (Hymenoptera: Aphelinidae). Int. J. of Insect Morphology and Embryology 23: 169-172.

Zchori-Fein, E., O. Faktor, M. Zeidan, Y. Gottlieb, H. Czosnek, and D. Rosen. 1995. Parthenogenesis-inducing microorganisms in Aphytis (Hymenoptera: Aphelinidae). Insect Molecular Biology 4:173-178.

\section{Patents: None}


